\title{
Spatially correlated disorder in self-organized precursor magnetic nanostructures
}

\author{
Marcel Porta, ${ }^{1,2}$ Teresa Castán, ${ }^{1,2}$ Pol Lloveras, ${ }^{1,2}$ Antoni Planes, ${ }^{1,2}$ and Avadh Saxena ${ }^{2,3}$ \\ ${ }^{1}$ Departament d'Estructura i Constituents de la Matèria, Universitat de Barcelona, Diagonal 647, 08028 Barcelona, Catalonia, Spain \\ ${ }^{2}$ Institut de Nanociència i Nanotecnologia de la Universitat de Barcelona, 08028 Barcelona, Catalonia, Spain \\ ${ }^{3}$ Theoretical Division, Los Alamos National Laboratory, Los Alamos, New Mexico 87545, USA \\ (Received 4 December 2006; revised manuscript received 22 June 2007; published 20 August 2007)
}

\begin{abstract}
We study the scaling behavior of the characteristic length of precursor magnetic nanostructures above the Curie temperature with the correlation length of quenched-in disorder. We found that the modulation length of the nanostructures $\xi$ follows the scaling law $\xi \sim \sqrt{\bar{D}}$, where $\bar{D}$ is the average size of the magnetized regions in the material. The scaling behavior of the average size of these regions, $\bar{D}$, with the correlation length of the disorder, $\sigma$, depends on the properties of the disorder. For Gaussian disorder, we find that $\bar{D}$ scales with the disorder correlation length as $\bar{D} \sim \sigma^{a / 2}$, where $a$ is the exponent of the leading term of the pair correlation function of the disorder in the limit $r \rightarrow 0, \Gamma(r) \approx \varepsilon\left[1-(1 / a)(r / \sigma)^{a}\right]$. These results are quite general and applicable to other systems, e.g., ferroelectric precursors, independent of the nature of the long-range dipolar forces.
\end{abstract}

DOI: 10.1103/PhysRevB.76.054432

PACS number(s): 75.30.Kz, 75.40.Mg, 61.43.-j

\section{INTRODUCTION}

Self-organized nanostructures are of considerable interest because of their potential importance in engineering functional materials. ${ }^{1}$ A peculiar situation is that of modulated nanoscale textures which originate as precursors to phase transitions in multiferroic materials. Such textures have been revealed by high-resolution imaging techniques well above the phase transition. ${ }^{2}$ This type of precursor $^{3}$ was first observed in the case of ferroelastic structural transitions with modulations in strain. The corresponding pattern exhibits anisotropic cross-hatched correlations that in real-space straincontrast images resemble the tweed textile. ${ }^{4}$ More recently, ${ }^{5}$ precursor structures with modulations in the magnetization giving rise to stripelike patterns have been observed in the $\mathrm{Co}_{0.38} \mathrm{Ni}_{0.33} \mathrm{Al}_{0.29}$ magnetic alloy above the Curie point. This led to the suggestion ${ }^{6}$ that the tweed concept is not just structural but applicable to a much broader class of materials with modulations in other physical variables (strain, magnetization, polarization). In addition, it was shown $\mathrm{n}^{6,7}$ that the origin of tweed lies in very general requirements, likely to be satisfied in quite different systems undergoing phase transitions. In short, the tweedlike modulations occurring above a phase transition are the natural global response of anisotropic longrange dipolar forces (elastic, magnetic, electric) to local perturbations arising from quenched-in disorder coupling to the order parameter (strain, magnetization, polarization).

The natural source for disorder in (magnetic) alloys is statistical compositional fluctuations that are quenched in during the alloying process. Thus, the alloy composition is an inhomogeneous quantity that is spatially correlated in order to avoid drastic variations from one point to another. In general one does not expect such correlations to be long ranged. Nevertheless, the spatial inhomogeneities might induce long-range interactions (e.g., elastic) that mediate the correlations.

Here we shall give special attention to precursor modulations in magnetic alloys. The modulations in the magnetization above the Curie point ${ }^{5}$ occur at a scale $\sim 100 \mathrm{~nm}$ and as mentioned above the pattern is stripelike with stripes being either vertical or horizontal due to magnetic anisotropy. In a previous work, ${ }^{7}$ we demonstrated that the intermediate tweedlike regime does exist and corresponds to a paramagnetic textured phase with modulations occurring at a length scale smaller than the magnetic domains observed below the Curie point. In the dipolar (or ferromagnetic) phase, the size of the stripes (i.e., the modulation $\xi$ ) follows the standard ${ }^{8}$ scaling law given by $\xi \sim \sqrt{L}$, where $L$ denotes the crystal size. In the (intermediate) precursor phase the modulations of the magnetization are localized in certain regions of the material of size $D \ll L$. Then the modulation length $\xi$ inside these regions should depend on $D$, which in turn depends on the correlation length of the disorder, $\sigma$. In the present paper we use Gaussian disorder and study the scaling behavior of both $D$ and $\xi$ for different functional forms of the pair correlation function of the disorder $\Gamma(r)$.

We shall first focus on the fundamental aspects of the problem and later discuss potential practical implications. The paper is organized as follows. In the next section we present a model for a two-dimensional paramagnetic to ferromagnetic transition with magnetic tweed present above the Curie temperature in the presence of quenched-in disorder and long-range magnetic dipolar interaction. Section III contains the numerical results obtained for the scaling behavior with $\sigma$ of both the size of the magnetized regions, $D$, and the stripe modulation length $\xi$. This is done for different (stretched) exponential $\Gamma(r)$ functions. In Sec. IV we focus on the statistical properties of the disorder itself and provide a theoretical analysis to link such results to the problem of interest here. In the next section (Sec. V) we also discuss the scaling behavior of the stripe modulation length in the dipolar and paramagnetic textured phases. Finally, in Sec. VI, we summarize our main findings and discuss their relevance in the context of engineering nanoscale functional materials. Some technical details pertinent to the pair correlation function $\Gamma(r)$ are relegated to Appendixes A and B. 

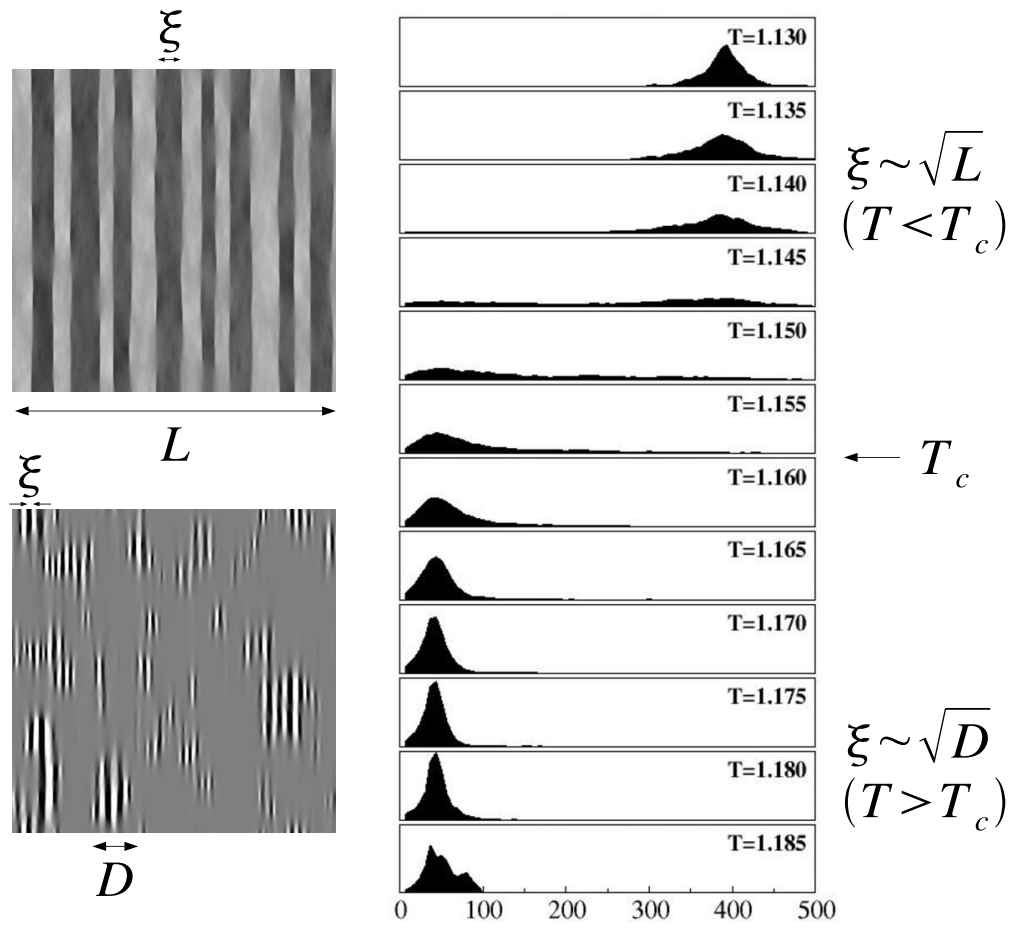

FIG. 1. Stripe modulation length distribution at different temperatures $\left(T_{c}=1.157\right)$. The phase transition from the dipolar to the paramagnetic textured phase is revealed in the scaling behavior, which changes from $\xi \sim \sqrt{L}$ below the Curie temperature to $\xi \sim \sqrt{D}$ in the precursor phase above the Curie temperature. The panels on the left depict the representative magnetization patterns in the two cases.

\section{THE MODEL}

The model we use is based on a mesoscopic GinzburgLandau description in two dimensions, extended to account for anisotropic long-range interactions necessary to produce large-scale effects in response to local perturbations (e.g., quenched disorder, inhomogeneities). The model has been discussed in detail previously. ${ }^{7}$ Here we summarize only briefly, recalling the key aspects relevant for the present study.

The magnetic free energy is the summation of three different contributions,

$$
F=F_{L}+F_{\text {grad }}+F_{\text {dip }}
$$

where the Landau free energy $F_{L}$ is a double-well potential in the magnetization, usual for continuous phase transitions:

$$
F_{L}=\int\left\{A\left[T-T_{c}^{0}(\mathbf{r})\right] m^{2}(\mathbf{r})+B m^{4}(\mathbf{r})+f_{A}(\mathbf{m})\right\} d \mathbf{r}
$$

Here $f_{A}(\mathbf{m})$ is the magnetocrystalline anisotropy. Motivated by the experiments, ${ }^{5}$ this term is taken to be uniaxial. Additionally, this choice is particularly convenient for computer simulations since it avoids competition between the two types of stripes possible in a square lattice. The statistical quenched-in disorder is introduced through a local coupling to the magnetization in the harmonic term. It has an effect on the transition temperature ${ }^{9} T_{c}^{0}(\mathbf{r})=T_{c}^{0}+\eta(\mathbf{r})$, which now locally fluctuates in space around $T_{c}^{0}$. Here $\eta(\mathbf{r})$ is a Gaussian disorder ${ }^{10}$ with zero mean and pair correlation function $\Gamma(r)$. The Ginzburg term $F_{\text {grad }}$ is the (short-range) gradient term that induces multiscale competition and has the following expression:

$$
F_{\text {grad }}=\kappa_{\text {grad }} \int d \mathbf{r} \sum_{i, k=x, y}\left(\frac{\partial m_{i}(\mathbf{r})}{\partial r_{k}}\right)^{2} .
$$

Finally, $F_{\text {dip }}$ is the two-dimensional long-range dipolar magnetic interaction that in the reciprocal space can be written ${ }^{6,7}$ as follows:

$$
\begin{aligned}
F_{\text {dip }}= & 2 \pi^{3} g \int d \mathbf{k} \frac{1}{k^{2}}\left\{( k _ { x } ^ { 2 } - k _ { y } ^ { 2 } ) \left[m_{x}(\mathbf{k}) m_{x}(-\mathbf{k})-m_{y}(\mathbf{k})\right.\right. \\
& \left.\left.\times m_{y}(-\mathbf{k})\right]+2 k_{x} k_{y}\left[m_{x}(\mathbf{k}) m_{y}(-\mathbf{k})+m_{y}(\mathbf{k}) m_{x}(-\mathbf{k})\right]\right\} .
\end{aligned}
$$

The conjunction of these three terms, given by Eqs. (2)-(4), produces a global response in terms of stripelike patterns both above and below the transition temperature.

\section{STRIPE MODULATION LENGTH BEHAVIOR: NUMERICAL RESULTS}

In a previous paper ${ }^{7}$ we showed that the precursor (tweedlike) phase corresponds to a paramagnetic textured phase. In other words, the averaged solution (over many disorder configurations) of the model in Sec. II keeps the symmetry of the high-temperature phase. Moreover, its characteristic stripe modulation length $\xi$ is smaller than in the (ferromagnetic) dipolar phase. This is illustrated in Fig. 1 from the modulation length distribution obtained at different temperatures (in reduced units) above and below the Curie point $\left(T_{c} \approx 1.157\right)$. The low-temperature peak (upper part) results from the modulation of the dipolar phase whereas the hightemperature peak (lower part) is related to the magnetic precursor modulation inside each region of width $D$. The size distribution abruptly changes at the Curie point and therefore the two modulated phases are clearly differentiated. 
As follows from a competition between the surface and the bulk energies, here we shall corroborate that the scaling behavior changes from the standard ${ }^{8} \xi \sim \sqrt{L}$ in the dipolar phase to $\xi \sim \sqrt{D}$ in the precursor phase.

It was suggested ${ }^{7,11}$ that in the precursor phase $\xi$ should depend on the disorder correlation length $\sigma$. Other a priori physically relevant lengths are the width of the domain walls $(W)$, and the size of the system $(L)$. From Fig. 1 one observes that the size of the magnetized regions $(D)$ is such that $W$ $\ll D \ll L$. Consequently, we expect that in this regime $\xi$ should scale with $D$, which in turn should depend on $\sigma$. From this perspective, we have performed numerical simulations and have checked that in the precursor phase $\xi$ does not depend on $L$, but it is sensitive to $\sigma$ and exhibits a clear scaling behavior $\xi \sim \sigma^{n}$ with scaling exponent $n$, which depends on the pair correlation function of the disorder. We have taken the following functional form for the pair correlation function: ${ }^{12}$

$$
\Gamma(r)=\varepsilon \exp \left[-\frac{1}{a}\left(\frac{r}{\sigma}\right)^{a}\right]
$$

where $\varepsilon$ is the strength of the disorder in reduced units and $\sigma$ is the corresponding correlation length. Numerical calculations ${ }^{13}$ have been performed on a simulation cell of size $l \ll L$ chosen to depend linearly on $\sigma$ as $l=12.5 \sigma$. Moreover, we have checked that the size of the mesh is sufficiently small in the sense that the numerical results do not depend on the discretization parameter. Calculations have been performed for three different values of the exponent $a$ $=2,1$, and $1 / 2$, and the averages have been taken over different realizations of the quenched-in disorder, the number ranging from 10 to 45 , depending on $l$. As we already mentioned above, $\xi$ does not depend on the system size $(L)$. $L$ is a physically relevant length scale of the problem and should not be confused with the size of the simulation cell, $l$, which is not a physical length, but it is one of the parameters of the simulation. It is introduced for computational purposes, and is at the origin of any finite-size effects arising in the present simulations. We shall return to this point later.

In Fig. 2(a), we show the behavior of the average size of the magnetized regions, $\bar{D} \equiv \sqrt{\left\langle D^{2}\right\rangle}$, with the correlation length of the disorder, $\sigma$. In Fig. 2(b), we have plotted the behavior of the stripe modulation length $\xi$ (inside the magnetized regions) with $\sigma$. Both lengths exhibit a power-law behavior; namely, $\bar{D} \sim \sigma^{m}$ and $\xi \sim \sigma^{n}$ with fitted exponents $m$ and $n$ summarized in Table I and the behavior shown with dashed lines in Fig. 2. The error bars have been estimated from the standard deviation of the distribution of values obtained by sampling over different subsets from the whole set of configurations. The results seem to be consistent with the scaling relations $\bar{D} \sim \sigma^{a / 2}$ and $\xi \sim \sigma^{a / 4}$, denoted by solid lines in Fig. 2. In the last column of Table I we have included the exponent $\beta$ for the scaling relation $\xi \sim \bar{D}^{\beta}$, obtained as $\beta$ $=n / m$. It naturally follows that $\xi \sim \sqrt{\bar{D}}$, in complete analogy with the scaling behavior $\xi \sim \sqrt{L}$ operative below the Curie point.

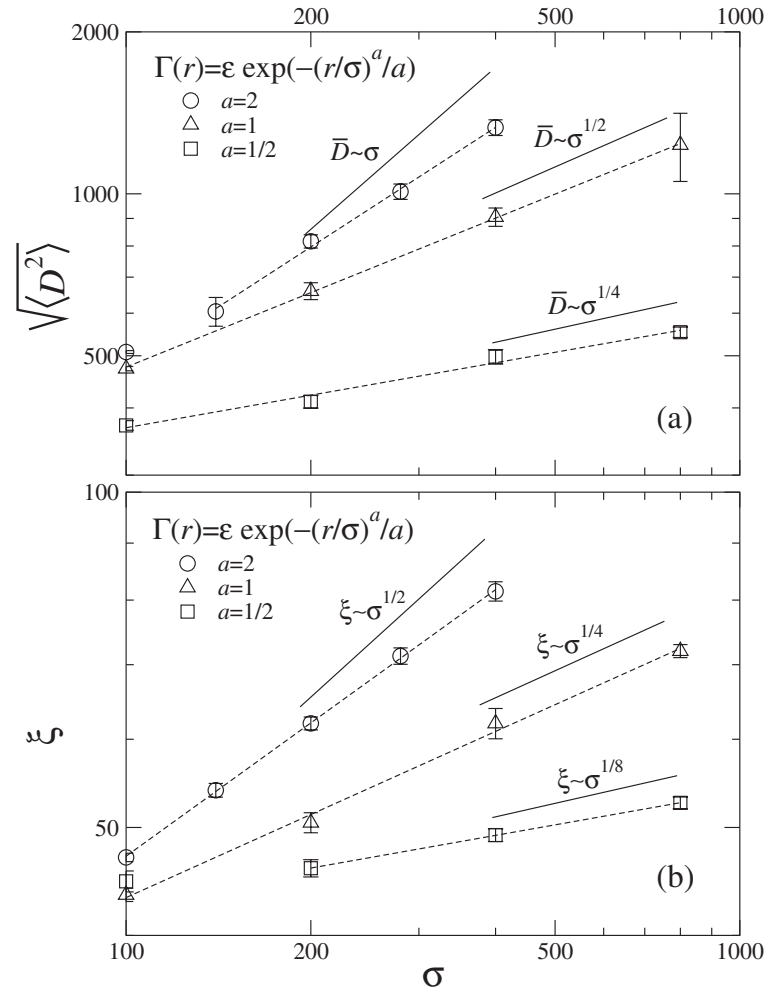

FIG. 2. Log-log plot showing the scaling dependence on the disorder correlation length $\sigma$ of (a) the mean size of the magnetized regions $\bar{D} \equiv \sqrt{\left\langle D^{2}\right\rangle}$ and (b) the magnetic stripe modulation length $\xi$, obtained from the position of the peak of the stripe modulation length distribution. In both cases, the scaling behavior has been obtained for three different values of $a=2$ (circles), 1 (triangles), and $1 / 2$ (squares). Power-law fits are shown as dashed lines, whereas solid lines correspond to the reference behavior $\bar{D} \sim \sigma^{a / 2}$ and $\xi \sim \sigma^{a / 4}$.

In the next section we shall provide theoretical evidence supporting the scaling relation $\bar{D} \sim \sigma^{a / 2}$. Systematic deviations from the relationship $m=2 n=a / 2$ may be attributed to the size of the simulation cell, $l$, as well as to a coupling to the width of the domain walls, $W$, for small values of $\sigma$. We recall that the simulation cell has been taken to be of variable size according to $l=12.5 \sigma$. This was done in order to minimize finite-size effects on the $\sigma$ dependence of both $\bar{D}$ and $\xi$. By looking at results in Table I, one observes that for $a=2$ and $a=1 / 2$, the deviation of the scaling exponent is about $20-25 \%$, whereas for $a=1$ it is less than $10 \%$. Again, we suggest that this is related to finite-size effects (for $a=2$ ) and to interference of $\bar{D}$ (or $\xi$ ) with $W$ (for $a=1 / 2$ ).

TABLE I. Scaling exponents obtained from a power-law fit to the numerical data (see text).

\begin{tabular}{lccc}
\hline \hline$a$ & $m$ & $n$ & $\beta=n / m$ \\
\hline 2 & $0.74 \pm 0.08$ & $0.40 \pm 0.02$ & 0.54 \\
1 & $0.46 \pm 0.04$ & $0.25 \pm 0.02$ & 0.54 \\
$1 / 2$ & $0.20 \pm 0.02$ & $0.10 \pm 0.02$ & 0.50 \\
\hline \hline
\end{tabular}



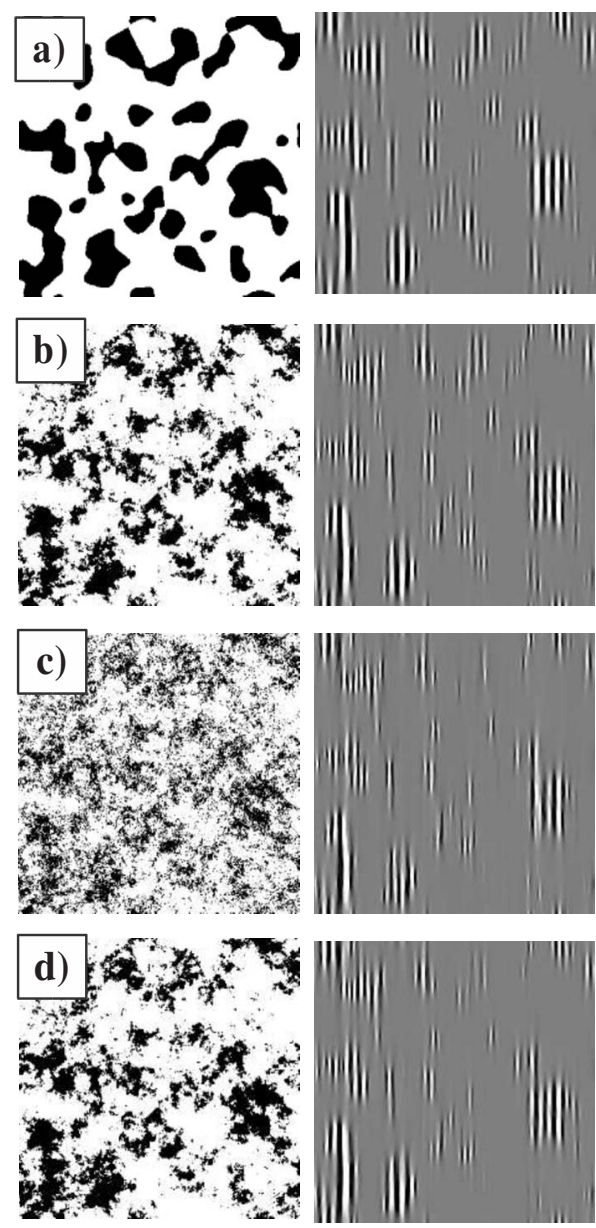

FIG. 3. Left column: Regions where $\nu(\mathbf{r})=+1$ (in black) for the stretched exponential $\Gamma(r)$ with (a) $a=2$, (b) 1 (usual exponential), and (c) $1 / 2$, and (d) for the power-law $\Gamma(r)$ with $a=1$. All cases shown correspond to a single realization of the disorder generated with the same sequence of random numbers. Right column: Corresponding magnetization patterns obtained after minimization of the total free energy.

\section{SPATIALLY CORRELATED DISORDER: SCALING BEHAVIOR}

In the precursor phase, the most likely regions to be magnetized are those where the local transition temperature $\left[T_{c}(\mathbf{r})\right]$ is above the actual temperature $T$. Consequently, the statistical properties of the magnetized regions $D$ are strongly related to the properties of the underlying static disorder. With this in mind, we proceed by investigating the properties of the disorder itself. To this end, we define a discrete local variable $\nu(\mathbf{r}) \equiv \operatorname{sgn}\left[T_{c}(\mathbf{r})-T\right]$ where $^{9} T_{c}(\mathbf{r})$ $=T_{c}+\eta(\mathbf{r})$ is a (continuous) Gaussian disorder distributed around $T_{c}$. The two values of $\nu(\mathbf{r})$ denote local transition temperatures above or below $T$. In the left column of Fig. 3 we have depicted in black the regions where $\nu(\mathbf{r})=+1$, for two generic types of pair correlation function; namely, the (stretched) exponential ${ }^{12}$ [Eq. (5)] and the power law, defined as

$$
\Gamma(r)=\frac{\varepsilon}{\left[1+(1 / 2)(r / \sigma)^{a}\right]^{2 / a}} .
$$

Again, $\varepsilon$ is the strength of the disorder in reduced units and $\sigma$ is the corresponding correlation length. Figures 3(a)-3(c) correspond to the same (stretched) exponential correlation functions used in the previous section (see Fig. 2) with $a$ $=2,1$, and $1 / 2$, respectively. It becomes clear that the exponent $a$ controls the degree of clustering of the regions where $\nu(\mathbf{r})=+1$, ranging from compact regions for $a=2$ to highly dispersed regions for $a=1 / 2$. In the last case Fig. 3(d) $\Gamma(r)$ is of the power-law type with $a=1$. In all cases the value of the correlation length is the same $(\sigma=200)$. We notice that in order to reveal differences arising from $\Gamma(r)$ only, the disorder in all cases shown in Fig. 3 has been generated using the same sequence of random numbers. For this reason, although the degree of clustering of the regions where $\nu(\mathbf{r})=+1$ is different from one snapshot to another, the spatial distribution of these regions is similar in all cases. Simultaneously, and for illustration purposes only, in the right column we show the corresponding magnetization patterns obtained after minimization of the total free energy [Eq. (1)], when considering the coupling of the disorder to the magnetization in Eq. (2). Notice that the snapshots in the left column reflect the underlying disorder map responsible for the response in terms of precursor modulated patterns shown in the right column. In spite of the differences observed in the shapes and sizes (and the degree of clustering) of the regions where $\nu(\mathbf{r})=+1$, the self-organized structures appear to be very robust with regard to the details of the pair correlation function. This is an important signature that the global response of the magnetic system is governed by the long-range interaction.

Let us denote by $\langle\lambda\rangle$ the mean size of regions where $\nu(\mathbf{r})=+1$ and study the corresponding scaling behavior with the correlation length of the disorder, $\sigma$. Notice that both $\langle\lambda\rangle$ and $\sigma$ are properties of the disorder only. We denote by $\Gamma_{\nu}(r)=\left\langle\nu\left(\mathbf{r}^{\prime}\right) \nu\left(\mathbf{r}^{\prime}+\mathbf{r}\right)\right\rangle$ the pair correlation function of the discrete variable $\nu(\mathbf{r})$. It is possible to show that $\langle\lambda\rangle$ is related to the derivative of $\Gamma_{\nu}(r)$ around $r \rightarrow 0$. This is detailed in Appendix A and we get

$$
\langle\lambda\rangle=-2 \frac{1+\langle\nu\rangle}{\left.\frac{d \Gamma_{\nu}(r)}{d r}\right|_{r \rightarrow 0}} .
$$

Moreover, the discrete pair correlation function $\Gamma_{\nu}(r)$ can be related to its continuous counterpart $\Gamma(r)$ in the case ${ }^{14}$ of $\langle\nu\rangle=0$ (see Appendix B for details),

$$
\Gamma_{\nu}(r)=\frac{2}{\pi} \arcsin \left(\frac{\Gamma(r)}{\varepsilon}\right) .
$$

Recall that $\Gamma(r)$ is the pair correlation function of the continuous variable $\eta(\mathbf{r})$, which describes the statistical distribution of local transition temperatures. By expanding $\Gamma(r)$ around $r=0$, one obtains ${ }^{15}$ that $\Gamma(r) \approx \varepsilon\left[1-(1 / a)(r / \sigma)^{a}\right]$ and its derivative 


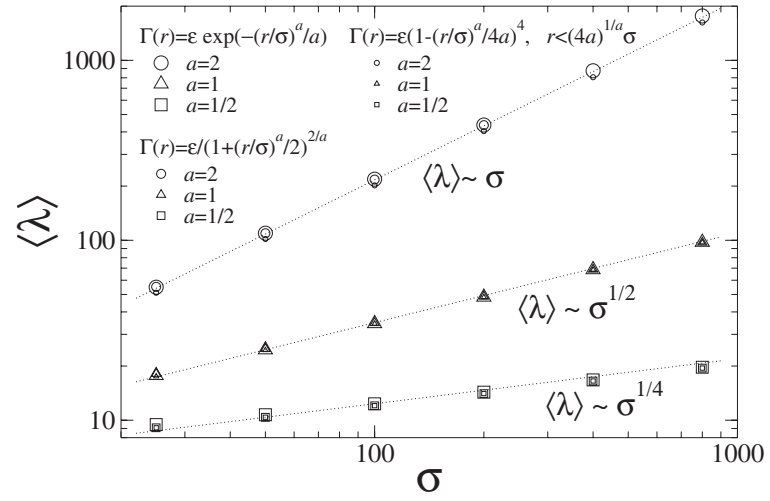

FIG. 4. Log-log plot showing the average size of the regions $\langle\lambda\rangle$, where $\nu(\mathbf{r})=+1$, as a function of the disorder correlation length $\sigma$ (in reduced units). The results correspond to an average over 200 configurations of disorder.

$$
\left.\frac{d \Gamma(r)}{d r}\right|_{r \rightarrow 0} \approx-\frac{\varepsilon}{\sigma}\left(\frac{r}{\sigma}\right)^{a-1}
$$

Then, differentiating both sides of Eq. (8), we get

$$
\frac{d \Gamma_{\nu}(r)}{d r}=\frac{2}{\pi} \frac{1}{\sqrt{\varepsilon^{2}-\Gamma^{2}(r)}} \frac{d \Gamma(r)}{d r},
$$

and introducing (9) into (10), it reads

$$
\left.\frac{d \Gamma_{\nu}(r)}{d r}\right|_{r \rightarrow 0} \approx-\frac{\sqrt{2 a}}{\pi \sigma}\left(\frac{r}{\sigma}\right)^{a / 2-1} .
$$

Finally, using the expression for $\langle\lambda\rangle$ in (7) with $\langle\nu\rangle=0$, we obtain that

$$
\langle\lambda\rangle \approx \pi \sigma \sqrt{\frac{2}{a}}\left(\frac{r}{\sigma}\right)^{1-a / 2} .
$$

For $a=2,\langle\lambda\rangle \sim \sigma$. In any other case $(a<2),\langle\lambda\rangle \rightarrow 0$ as $r$ $\rightarrow 0$. Nevertheless, if the space is discretized there is a minimum size of $\lambda$ which corresponds to the size of the mesh, $\Lambda$. In such a case, we obtain

$$
\langle\lambda\rangle=-\frac{2 \Lambda}{\Gamma_{\nu}(\Lambda)-1} \approx \pi \sigma \sqrt{\frac{a}{2}}\left(\frac{\Lambda}{\sigma}\right)^{1-a / 2},
$$

and we might write

$$
\langle\lambda\rangle \sim \Lambda^{1-a / 2} \sigma^{a / 2} \sim \sigma^{a / 2} .
$$

To check the expression above, we have performed extensive calculations for different values of $\sigma$ and using different functional forms for $\Gamma(r)$; namely, (stretched) exponential [Eq. (5)] and power law [Eq. (6)], both defined previously, and a polynomial. The last one is defined as

$$
\Gamma(r)= \begin{cases}\varepsilon\left[1-\frac{1}{4 a}\left(\frac{r}{\sigma}\right)^{a}\right]^{4}, & r<(4 a)^{1 / a} \sigma, \\ 0, & r \geqslant(4 a)^{1 / a} \sigma .\end{cases}
$$

The results are shown in a log-log plot in Fig. 4. For all cases considered, we obtain that $\langle\lambda\rangle \sim \sigma^{a / 2}$ with high accuracy, in agreement with the theoretical results given above. More- over, we notice that the numerical results correspond to $T$ $=1.165>T_{c}$, extending the validity of Eq. (14) to $\langle\nu\rangle \neq 0$.

We now return to our magnetic problem. In Sec. III we obtained numerically that the scaling behavior of the mean size of the magnetized regions is consistent with $\bar{D} \sim \sigma^{a / 2}$. Then, it follows that there should be a linear relation between $\langle\lambda\rangle$ and $\bar{D}$,

$$
\bar{D} \sim\langle\lambda\rangle \sim \sigma^{a / 2} .
$$

The largest discrepancy between the numerical results and the scaling laws given in Eq. (16) lies in the relation $\bar{D}$ $\sim \sigma^{a / 2}$ (see Table I), which arises from the assumption of a linear relation between $\bar{D}$ and $\langle\lambda\rangle$. As discussed in Sec. III, this deviation is attributed to finite-size effects and to coupling to $W$. In any case, we remark that both effects are similar on either $\bar{D} \sim \sigma^{m}$ or $\xi \sim \sigma^{n}$, so that the relation $\xi$ $\sim \sqrt{\bar{D}}$ is satisfied in all cases with very good accuracy.

\section{MODULATION LENGTH: SCALING BEHAVIOR}

From the analysis of the results presented in the previous sections it follows that above the Curie point, in the precursor phase, the relevant length for the scaling behavior of the modulation length $\xi$ is the disorder correlation length $\sigma$ $\gg W$, where $W$ is the width of the domain walls. In this intermediate regime the modulation $\xi$ occurs inside a magnetized region of size $D$ so that $W \ll \xi \approx \sigma \approx \bar{D} \ll L$ and $\xi$ $\sim \sqrt{\bar{D}} \sim \sigma^{n}$. The scaling exponent $n$ depends on the leading term of the pair correlation function $\Gamma(r)_{r \rightarrow 0} \sim r^{a}$. We find that $n=a / 4$.

As the temperature decreases toward $T_{c}$, the size of the magnetized regions $D$ increases and the magnetization pattern collapses at $T=T_{c}$. From that point, $\sigma \ll \bar{D} \approx L$ and then it follows that $\xi \sim \sqrt{L}$. We notice that in both regimes (see the representative patterns in the left column of Fig. 1) the scaling dependence of the stripe modulation depends on the average size of the magnetized regions ("modulated droplets"). This behavior is dictated by thermodynamic arguments, i.e., a balance between the long-range dipolar interaction and the short-range domain wall energy. ${ }^{8}$ We also notice that our numerical results are not based on the scaling relation $\xi$ $\sim \sqrt{\bar{D}}$ but they are fully consistent with it.

\section{CONCLUSION}

Magnetic tweed refers to modulations in the magnetization above the Curie temperature. In a magnetic alloy such as $\mathrm{Co}_{0.38} \mathrm{Ni}_{0.33} \mathrm{Al}_{0.29}$ it is caused by intrinsic statistical compositional fluctuations (or static disorder), which lead to a local Curie temperature that fluctuates from one point to another. We have systematically studied the scaling behavior of the mean size of the magnetized regions, $\bar{D}$, and the stripe modulation length $\xi$ as a function of the form of the disorder correlation function $\Gamma(r)$ and disorder correlation length $\sigma$ in magnetic alloys ${ }^{5}$ exhibiting the precursor phenomenon above 
the Curie temperature. We found that $\bar{D} \sim \sigma^{m}$ and $\xi \sim \sigma^{m / 2}$, where the exponent $m$ depends only on the exponent $a$ of the leading term in $\Gamma(r)$ as $r \rightarrow 0$ but not on the specific functional form of $\Gamma(r)$. Deviations from the relationship $m$ $=a / 2$ are attributed to interference with other lengths in the problem, namely, the domain wall width $W$ and the simulation cell size $l$. Although $l$ is not a physical length, all the results are affected by finite-size effects (especially for large $\bar{D})$ due to the impossibility of simulating the whole system (of size $L$ ), which from a practical point of view is infinite.

There are interesting implications of our results. In particular, they are important in controlling the length scale of self-organized modulated structures. Alternatively, it appears possible to engineer nanoscale magnetic functionalities in a material. One can envision a specific nanoscale disorder patterning of a magnetic alloy by controllably implanting impurities with a given $\sigma$ of the disorder. Conversely, by measuring the size distribution of the magnetized regions or the stripe modulation lengths (see the right panel of Fig. 3), it may be possible to infer the disorder correlation length $\sigma$. In addition, the model is quite general and applicable to both polar as well as ferroelectric tweed. ${ }^{16}$ The former is the ana$\log$ of the magnetic tweed discussed here, whereas the latter is analogous to magnetoelastic tweed. Indeed, it would be desirable to study the scaling properties of the magnetoelastic tweed ${ }^{17}$ observed in $\mathrm{Co}_{2} \mathrm{NiGa}, \mathrm{Ni}_{2} \mathrm{FeGa}$, and $\mathrm{Ni}_{2} \mathrm{MnGa}$ alloys. Recent imaging studies of magnetoelastic tweed exhibit its coupling to the structural tweed and its contrast is found to be orientation dependent. ${ }^{18}$

\section{ACKNOWLEDGMENTS}

This work was supported by CICyT (Spain) Project No. MAT2004-01291, Marie-Curie RTN MULTIMAT (Contract No. MRTN-CT-2004-505226), DURSI (Catalonia) Project No. 2005SGR00969 and the U.S. Department of Energy. M.P. is thankful for the hospitality of the Theoretical Division at Los Alamos National Laboratory.

\section{APPENDIX A}

In this appendix we derive an analytical expression for the mean size of the regions where $\nu(\mathbf{r})=+1$ as a function of the mean value of the disorder $\nu(\mathbf{r})= \pm 1$, and its corresponding pair correlation function $\Gamma_{\nu}(r)=\left\langle\nu\left(\mathbf{r}^{\prime}\right) \nu\left(\mathbf{r}^{\prime}+\mathbf{r}\right)\right\rangle$, which is assumed to be isotropic.

Let us define $P(r)$ as the probability that $\nu\left(\mathbf{r}^{\prime}+\mathbf{r}\right)=+1$ on the condition that $\nu\left(\mathbf{r}^{\prime}\right)=+1$. The conditional probability $P(r)$ is related to the pair correlation function of the disorder $\nu(\mathbf{r})$, that is, $\Gamma_{\nu}(r)$, by the following expression:

$$
P(r)=1-\frac{1}{2} \frac{1-\Gamma_{\nu}(r)}{1+\langle\nu\rangle} .
$$

If $\nu\left(\mathbf{r}^{\prime}\right)=+1$ and the size (along the $\mathbf{r}$ direction) of the region that contains $\mathbf{r}^{\prime}$ is $\lambda>r$, the probability that $\nu\left(\mathbf{r}^{\prime}+\mathbf{r}\right)=+1$ can be approximated by $(\lambda-r) / \lambda$, that is, the probability that the distance between $\mathbf{r}^{\prime}$ and the edge of the region of size $\lambda$, where $\mathbf{r}^{\prime}$ is included, is larger than $r$. This is an approximation that will be valid only for small values of $r$, since we are neglecting the probability when $\mathbf{r}^{\prime}$ and $\mathbf{r}$ belong to different regions of $\nu=+1$.

Next, we define $\varphi(\lambda)$ as the probability density of the size of the regions where $\nu(\mathbf{r})=+1$. The probability density that the position $\mathbf{r}^{\prime}$ belongs to a region of size $\lambda$ is therefore,

$$
\Phi(\lambda)=\frac{\lambda \varphi(\lambda)}{\int_{0}^{\infty} \lambda \varphi(\lambda) d \lambda}=\frac{\lambda \varphi(\lambda)}{\langle\lambda\rangle} .
$$

Thus, the probability that $\nu\left(\mathbf{r}^{\prime}+\mathbf{r}\right)=+1$ on the condition that $\nu\left(\mathbf{r}^{\prime}\right)=+1$, that is, $P(r)$, can be approximated by

$$
P(r) \approx \int_{r}^{\infty} \Phi(\lambda)\left(\frac{\lambda-r}{\lambda}\right) d \lambda=\frac{1}{\langle\lambda\rangle} \int_{r}^{\infty} \varphi(\lambda)(\lambda-r) d \lambda .
$$

Differentiating the conditional probability, for $r \rightarrow 0$ we obtain

$$
\left.\frac{d P(r)}{d r}\right|_{r \rightarrow 0} \approx-\frac{1}{\langle\lambda\rangle} \int_{0}^{\infty} \varphi(\lambda) d \lambda=-\frac{1}{\langle\lambda\rangle} .
$$

Therefore, in terms of the pair correlation function $\Gamma_{\nu}(r)$, the mean size of the regions where $\nu(\mathbf{r})=+1$ can be written as

$$
\langle\lambda\rangle=-2-\frac{1+\langle\nu\rangle}{\left.\frac{d \Gamma_{\nu}(r)}{d r}\right|_{r \rightarrow 0}} .
$$

If the space is discretized into a mesh of size $\Lambda$, the same arguments hold, and we get

$$
\langle\lambda\rangle=-2 \frac{1+\langle\nu\rangle}{\Gamma_{\nu}(\Lambda)-1} \Lambda .
$$

\section{APPENDIX B}

In this appendix we obtain the relationship between the pair correlation function of the discrete variable $\nu(\mathbf{r})$ at $T$ $=T_{c}$, denoted by $\Gamma_{\nu}(r)$, and $\Gamma(r)$ corresponding to the continuous variable $\eta(\mathbf{r})$.

The $N$-point probability density of the Gaussian disorder is a known Gaussian function. ${ }^{19}$ In particular, the two-point probability density of the Gaussian disorder with zero mean and isotropic pair correlation function $\Gamma(r)$ is

$$
\begin{aligned}
\rho\left(t, t^{\prime}, r\right)= & \frac{1}{2 \pi \sqrt{\varepsilon^{2}-\Gamma^{2}(r)}} \exp \left(-\frac{1}{2} \frac{\varepsilon}{\varepsilon^{2}-\Gamma^{2}(r)}\left(t^{2}+t^{\prime 2}\right)\right. \\
& \left.+\frac{\Gamma(r)}{\varepsilon^{2}-\Gamma^{2}(r)} t t^{\prime}\right),
\end{aligned}
$$

where $\varepsilon=\Gamma(0)$ is the strength of the disorder.

At $T=T_{c}, \nu(\mathbf{r})=\operatorname{sgn}[\eta(\mathbf{r})]$, and the pair correlation function $\Gamma_{\nu}(r)$ can be written as 


$$
\Gamma_{\nu}(r)=\left\langle\nu\left(\mathbf{r}^{\prime}\right) \nu\left(\mathbf{r}^{\prime}+\mathbf{r}\right)\right\rangle=\int_{0}^{\infty} d t \int_{0}^{\infty} d t^{\prime} \rho\left(t, t^{\prime}, r\right)-\int_{0}^{\infty} d t \int_{-\infty}^{0} d t^{\prime} \rho\left(t, t^{\prime}, r\right)-\int_{-\infty}^{0} d t \int_{0}^{\infty} d t^{\prime} \rho\left(t, t^{\prime}, r\right)+\int_{-\infty}^{0} d t \int_{-\infty}^{0} d t^{\prime} \rho\left(t, t^{\prime}, r\right),
$$

which leads to

$$
\Gamma_{\nu}(r)=\frac{2}{\pi} \arctan \left(\frac{\Gamma(r)}{\sqrt{\varepsilon^{2}-\Gamma^{2}(r)}}\right)=\frac{2}{\pi} \arcsin \left(\frac{\Gamma(r)}{\varepsilon}\right) .
$$

${ }^{1}$ N. Mathur and P. Littlewood, Nat. Mater. 3, 207 (2004).

${ }^{2}$ T. Castán, E. Vives, Ll. Mañosa, A. Planes, and A. Saxena, in Magnetism and Structure in Functional Materials, edited by A. Planes, Ll. Mañosa, and A. Saxena, Springer Series in Materials Science Vol. 79 (Springer-Verlag, Heidelberg, 2005), pp. 27-48, and references therein.

${ }^{3}$ L. E. Tanner, Philos. Mag. 14, 111 (1966).

${ }^{4}$ See, e.g., S. M. Shapiro, J. Z. Larese, Y. Noda, S. C. Moss, and L. E. Tanner, Phys. Rev. Lett. 57, 3199 (1986).

${ }^{5}$ Y. Murakami, D. Shindo, K. Oikawa, R. Kainuma, and K. Ishida, Acta Mater. 50, 2173 (2002).

${ }^{6}$ A. Saxena, T. Castán, A. Planes, M. Porta, Y. Kishi, T. A. Lograsso, D. Viehland, M. Wuttig, and M. De Graef, Phys. Rev. Lett. 92, 197203 (2004).

${ }^{7}$ M. Porta, T. Castán, A. Planes, and A. Saxena, Phys. Rev. B 72, 054111 (2005).

${ }^{8}$ C. Kittel, Rev. Mod. Phys. 21, 541 (1949).

${ }^{9}$ Actually, the transition temperature of the model depends on both the disorder and the strength of the dipolar interaction through $T_{c}(\mathbf{r})=T_{c}^{0}+\pi g / 2 A+\eta(\mathbf{r})$; see Eqs. (2) and (4).

${ }^{10}$ The Gaussian disorder has been generated using the algorithm described in J. García-Ojalvo and J. M. Sancho, Noise in Spatially Extended Systems (Springer, New York, 1999), p. 92.
${ }^{11}$ M. Porta, T. Castán, P. Lloveras, A. Planes, and A. Saxena, J. Magn. Magn. Mater. 310, 2641 (2006).

${ }^{12}$ This is called a stretched exponential and reduces to the usual exponential when $a=1$. S. H. Chung and J. R. Stevens, Am. J. Phys. 59, 1024 (1991); G. F. Signorini, J. L. Barrat, and M. L. Klein, J. Chem. Phys. 92, 1294 (1990).

${ }^{13}$ Details on the numerical implementation of the model are given in Ref. 7. The values of the parameters used here are $\varepsilon$ $=0.0015 / 2 \pi, T=1.165$, and $\Lambda=4.88$ (size of the mesh), in reduced units.

${ }^{14}$ Notice that $T=T_{c}$ means $\nu(\mathbf{r})=\operatorname{sgn}[\eta(\mathbf{r})]$.

${ }^{15}$ The leading term of the expansion of the pair correlation function $\Gamma(r)$ at $r=0$ is, by construction, the same for all the functional forms used.

${ }^{16}$ O. Tikhomirov, H. Jiang, and J. Levy, Phys. Rev. Lett. 89, 147601 (2002).

${ }^{17}$ H. S. Park, Y. Murakami, D. Shindo, V. A. Chernenko, and T. Kanomata, Appl. Phys. Lett. 83, 3752 (2003).

${ }^{18}$ S. P. Venkateswaran, N. T. Nuhfer, and M. De Graef, Acta Mater. 55, 2621 (2007).

${ }^{19}$ R. L. Stratonovich, Topics in the Theory of Random Noise (Gordon and Breach, New York, 1963), Vol. 1. 